\title{
Validação clínica de tecnologia educativa sobre prevenção do pé diabético
}

\author{
Clinical validation of educational technology on diabetic foot prevention \\ Validación clínica de tecnología educativa sobre prevención del pie diabético
}

Raquel Alves de Oliveira ${ }^{1 *}$, José Mateus Pires¹, Luisa Gomes Viana1, Mayara Maria Silva da Cruz Alencar ${ }^{1}$, João Victor Mendonça Santana Cavalcante ${ }^{1}$, Samila Gomes Ribeiro ${ }^{1}$, Priscila de Souza Aquino', Régia Christina Moura Barbosa Castro¹.

\section{RESUMO}

Objetivo: Validar clinicamente um álbum seriado sobre prevenção do pé diabético com usuários de um serviço de saúde. Métodos: Trata-se de um estudo metodológico, com uma amostra de 30 pacientes de uma unidade de saúde secundária especializada da capital cearense, que estavam em acompanhamento do Diabetes Mellitus e tratamento de suas complicações. Os dados foram coletados no mês de maio de 2019 por meio de entrevista contendo questões relacionadas às figuras da tecnologia em saúde. Foi utilizado o Índice de Validação do Conteúdo (IVC) para a validação do álbum, sendo as variáveis submetidas a teste estatístico de Normalidade de Shapiro-Wilk, adotando-se a mediana quando $\mathrm{p}<0,05$. A análise dos dados foi realizada por meio do software IBM SPSS Statistics. Resultados: Os critérios avaliados obtiveram valores superiores a 0,80 segundo o Índice de Validade de Conteúdo nos quatro domínios: organização, conteúdo, atratividade e persuasão, sendo os domínios "conteúdo" e "atratividade" os que apresentaram maior positividade nas respostas dos entrevistados. Conclusão: O álbum seriado foi validado clinicamente com boa aceitação pelos pacientes, representando uma tecnologia confiável e pertinente para utilização por profissionais da Saúde em todos os aspectos analisados.

Palavras-chave: Diabetes mellitus, Pé diabético, Educação em saúde, Enfermagem, Estudo de validação.

\begin{abstract}
Objective: To validate clinically a serial album about the prevention of diabetic foot with users of a health service. Methods: This is a methodological study with a sample of 30 patients from a specialized secondary Health Unit in the capital of Ceará, who were being monitored for Diabetes Mellitus and treatment of its complications. Data were collected in May 2019 by an interview with questions related to the pictures of the health technology. The Content Validity Index ( $\mathrm{CVI})$ was used to validate the album, and the variables were subjected to statistical Shapiro-Wilk's Normality test, adopting the median when $p<0.05$. Data analysis was performed using IBM SPSS Statistics software. Results: The evaluated criteria obtained values higher than 0.80 according to the Content Validity Index in the four domains: organization, content, attractiveness and persuasion, with the "content" and "attractiveness" domains being the most positive in the respondents' answers. Conclusion: The album has been clinically validated with good acceptance by patients, representing a reliable and relevant technology for the use by health professionals in all aspects analyzed.
\end{abstract}

Keywords: Diabetes mellitus, Diabetic foot, Health education, Nursing, Validation study.

\section{RESUMEN}

Objetivo: Validar clínicamente un álbum serial sobre la prevención del pie diabético con usuarios de un servicio de salud. Métodos: Se trata de un estudio metodológico, con una muestra de 30 pacientes de una unidad secundaria especializada de salud de la capital cearense, quienes estaban en seguimiento para Diabetes Mellitus y tratamiento de sus complicaciones. Los datos se recopilaron en mayo de 2019 a través de una entrevista que contenía preguntas relacionadas con cifras de la tecnología en Salud. Para la validación

${ }^{1}$ Universidade Federal do Ceará (UFC), Fortaleza - CE. *E-mail: raquelalvesgw@gmail.com 
del álbum se utilizó el Índice de Validez de Contenido (IVC), las variables fueron sometidas a prueba estadística de Normalidad de Shapiro-Wilk, adoptando la mediana cuando $p<0.05$. El análisis de los datos se realizó con el software IBM SPSS Statistics. Resultados: Los criterios evaluados obtuvieron valores superiores a 0,80 según el Índice de Validez de Contenido en los cuatro dominios: organización, contenido, atractivo y persuasión, siendo los dominios "contenido" y "atractivo" los más positivos en las respuestas de los encuestados. Conclusión: El álbum ha sido validado clínicamente con buena aceptación por parte de los pacientes, representando una tecnología confiable y relevante para su uso por parte de los profesionales de la salud en todos los aspectos analizados.

Palabras clave: Diabetes mellitus, Pie diabético, Educación en salud, Enfermería, Estudio de validación.

\section{INTRODUÇÃO}

O diabetes mellitus (DM) configura-se como um dos mais importantes problemas de saúde pública do século XXI, afetando cerca de $8,8 \%$ da população mundial, o que corresponde a 425 milhões de pessoas entre 20 e 79 anos de idade. Nesse contexto, o Brasil encontra-se em quarto lugar entre os dez países com maior prevalência da doença, com cerca de 12,5 milhões de pessoas acometidas (INTERNATIONAL DIABETES FEDERATION, 2017).

Dentre as complicações da diabetes, a neuropatia diabética é a de maior incidência a longo prazo, sendo as alterações sensoriais e motoras as de maior impacto na qualidade de vida dos pacientes, as quais podem levar a manifestação do pé diabético. Essa condição, que é decorrente da associação entre anormalidades neurológicas e vasculares, leva à perda da sensibilidade periférica térmica, tátil ou dolorosa, ocasionando a ulceração, infecção e destruição de tecidos, condição que, caso não corretamente tratada, pode levar a necessidade de amputação de pés e membros inferiores (FONSECA KP e RACHED CDA, 2019).

Um estudo realizado na cidade de Florianópolis/SC, constatou que mais de $60 \%$ dos indivíduos entrevistados não tiveram seus pés avaliados por profissional da saúde após ter recebido o diagnóstico de diabetes. Dessa forma, ressalta-se que muitos pacientes não recebem capacitação adequada no que diz respeito à prevenção e ao tratamento do pé diabético e de outras complicações da doença (BOELL JEW, et al., 2014).

Nesse contexto, é sabido que várias pesquisas estimulam a importância de haver um maior fornecimento de orientações acessíveis, bem como o incentivo ao autocuidado, visando à redução da ocorrência de complicações nos pés. Para isso, há necessidade de aproximação do profissional de saúde ao indivíduo, na posse de tecnologias cientificamente validadas para que o paciente esteja empoderado quanto à sua condição de saúde. Compreende-se que o uso de tecnologias educativas é positivo na apreensão do conhecimento, por meio de abordagem franca para que o indivíduo passe a tomar suas decisões com o enfoque na melhoria de sua condição (AQUINO JA, et al., 2016).

A utilização desses recursos em Educação em Saúde deve abranger o uso de tecnologias táteis e dialógicas para obter maior êxito na oferta de informação aos indivíduos (SANTOS CT, 2015). Assim, sua construção requer que elas abordem o conhecimento científico, o consenso de juízes e, principalmente, a análise crítica e a aprovação dos pacientes, visto que estes são o fim da construção da tecnologia educativa.

Um material educativo bem elaborado deve oferecer suporte para promover o senso crítico, explicitando dúvidas e discursos. Dessa forma, é importante inserir o público-alvo no processo de validação de materiais educativos, tendo em vista que outros estudos afirmam que é uma etapa relevante para o material a ser validado, onde ocorre a reformulação de informações e ilustrações, bem como a substituição de termos para o desenvolvimento de um instrumento de fácil compreensão e adequado para a realidade do público que utilizará o material (LIMA ACMACC, et al., 2017).

Dessa forma, acredita-se que as tecnologias são ferramentas criadas para ampliar as possibilidades dos profissionais de saúde para realizar práticas produtoras de cuidado e, consequentemente, melhorar a qualidade da assistência em saúde. Essas ferramentas são necessárias para que a construção do conhecimento seja de forma horizontalizada (BARBOSA EMG, et al., 2016). 
Nessa perspectiva, este estudo teve como objetivo validar clinicamente um álbum seriado sobre prevenção do pé diabético com usuários de um serviço de saúde.

\section{MÉTODOS}

Trata-se de um estudo metodológico, com centro na avaliação, pelo público-alvo, de um álbum seriado sobre prevenção do pé diabético. O álbum foi validado em 2018 com juízes de conteúdo e aparência, sendo cinco avaliadores especialistas na área de DM e dois especialistas em publicidade com enfoque no design gráfico. O álbum seriado conta, em sua versão final, com 14 páginas: a capa, onze figuras contendo a ficha roteiro para o profissional de saúde no verso das imagens, além da lista de identificação dos autores e a contracapa. O álbum é posicionado de forma que as ilustrações estejam visíveis para o paciente e as fichasroteiro fiquem à vista do aplicador do álbum.

A validação clínica do álbum "De olho na prevenção do pé diabético" contou com a participação voluntária de 30 pacientes portadores de DM que realizavam acompanhamento em uma unidade secundária de referência, especializada em atendimento a pacientes portadores de Hipertensão Arterial Sistêmica e DM no estado do Ceará. A unidade realiza atividades assistenciais aos pacientes durante o curso da doença e em suas complicações e conta com profissionais assistencialistas de várias áreas, como enfermeiros, médicos e profissionais de nível técnico.

Em relação ao tamanho amostral para a validação com o público-alvo, estima-se entre o mínimo de 25 e o máximo de 50 participantes (FEHRING R, 1986). Optou-se como coleta de dados, os pacientes atendidos no mês de maio de 2019.

Quanto aos critérios de inclusão participaram do estudo pacientes maiores de 18 anos que realizavam tratamento na referida unidade e aguardavam atendimento em sala de espera. Inicialmente, os pesquisadores responsáveis pela coleta dos instrumentos realizaram uma apresentação breve sobre o tema do álbum e a importância de sua abordagem. Em seguida, cada página do álbum seriado foi explicada aos pacientes. Foram coletados, por meio de entrevista, os dados sociodemográficos e clínicos dos participantes, bem como dados para validação do álbum seriado, consistindo em uma escala tipo Likert com os seguintes aspectos avaliados: clareza da linguagem, relevância e grau de relevância das páginas da cartilha (PASQUALI L, 2010).

Após o instrumento de validação, ocorreu avaliação de cada página do material educativo, a fim de preencher a ficha de avaliação composta por três perguntas: 1 . Esta figura lhe parece clara e compreensível?; 2. A composição visual está atrativa e bem organizada?; 3. Qual o grau de relevância da presença dessa figura no álbum? Para a pergunta de número 1, existiam três possibilidades: "Confuso", "pouco claro" ou "claro", já para o segundo questionamento, o participante poderia optar entre "não" ou "sim" e por fim, na pergunta de número 3 existiam quatro possibilidades: "irrelevante", "pouco relevante", "relevante" ou "muito relevante". O instrumento utilizado foi baseado em um estudo focado na elaboração e validação de uma cartilha educativa (SABINO LMM, et al, 2018).

Por fim, os participantes preenchiam um instrumento intitulado "Avaliação Geral do Álbum Seriado", dividido em cinco tópicos: organização, conteúdo, atratividade, aceitação cultural e persuasão, com três opções de resposta: "sim", "mais ou menos" e "não". Porém, o item de "aceitação cultural" era composto por perguntas abertas como: "O que você gostou no álbum?" e "O que você não gostou no álbum?".

O público alvo foi abordado durante o período de espera anterior a consulta. Após o momento inicial de apresentação dos pesquisadores, ocorreu a intervenção educativa com o álbum seriado de forma coletiva com duração de, aproximadamente, dez minutos. Posteriormente, a entrevista sobre os dados sociodemográficos e o material de validação foram aplicados de forma individual no mesmo ambiente de espera.

Para a validação de conteúdo foi utilizado o Índice de Validade de Conteúdo (IVC), que é baseado na seguinte equação matemática: proporção dos itens que receberam valores entre 3 e 4 (relevante e muito relevante). Para análise das variáveis numéricas foi utilizado o teste de Normalidade de Shapiro-Wilk e adotou-se a mediana quando $p<0,05$. Os achados foram apresentados por meio de tabelas e gráfico e 
discutidos de acordo com a literatura pertinente. A pesquisa foi aprovada pelo Comitê de Ética em Pesquisa da Maternidade Escola Assis Chateaubriand, sob número de parecer 1.739.998.

\section{RESULTADOS}

A sessão dos resultados foi dividida em três partes: caracterização sociodemográfica, clínica e dados relativos à validação com o público-alvo. Quanto à caracterização sociodemográfica, $16(53,3 \%)$ pacientes possuíam idade maior ou igual a 60 anos e 14 (46,7\%) encontravam-se na faixa etária de 25 a 59 anos.

A média de idade foi 58,2 anos, com desvio padrão de 15,9 anos. Desses indivíduos, 22 (73,3\%) eram do sexo feminino e $8(26,7 \%)$ do sexo masculino. Além disso, 14 (46,7\%) eram casados, $6(20 \%)$ eram solteiros e $4(16,7 \%)$ eram viúvos. Com relação à escolaridade, o período de 8 a 10 anos de estudo correspondeu ao maior quantitativo de indivíduos, totalizando 11 (36,7\%), com média de escolaridade de 8,62 anos e desvio padrão de 3,879 . A etnia representativa dessa amostra foi de $17(56,7 \%)$ pardas, $10(33,3 \%)$ brancas e 3 $(10 \%)$ pretas.

No que diz respeito à renda, 8 (40\%) participantes possuíam renda menor ou igual a um salário mínimo ( $R \$ 998,00$ à época da pesquisa), com mediana de 2 salários, variação de 1 a 3 e intervalo interquartil de 2. Em relação à residência, 16 (53,3\%) participantes moravam em Fortaleza. Já no que diz respeito à profissão, $9(30 \%)$ participantes eram aposentados, $4(13,3 \%)$ eram costureiras e $4(13,3 \%)$ estavam desempregados.

Quanto à caracterização clínica, destaca-se que o tempo de diagnóstico de diabetes foi 13 anos de mediana, com intervalo interquartil de 31, variando de 2 a 32 anos. Já no que diz respeito ao tempo de acompanhamento especializado, a mediana foi de 9,5 anos, com intervalo interquartil de 29 , variando de 1 a 30 anos. Ademais, com relação à via do tratamento medicamentoso, $24(80 \%)$ participantes apresentaram tratamento oral e $24(80 \%)$ tratamento injetável. Nas complicações e comorbidades associadas, a maioria dos indivíduos, $18(60 \%)$, apresentou hipoglicemia. Além disso, também foram relatadas presença de doença cardiovascular em $11(36,7 \%)$, perda de sensibilidade em $8(26,7 \%)$, neuropatia em $6(20 \%)$, cegueira em 5 $(16,7 \%)$ e retinopatia em $4(13,3 \%)$.

Com relação às orientações sobre pé diabético, $26(89,7 \%)$ pacientes relataram que receberam as orientações sobre o tema. A seguir, destaca-se a avaliação do álbum seriado quanto à organização, conteúdo, atratividade e persuasão (Tabela 1).

Tabela 1 - Distribuição das respostas positivas na avaliação dos pacientes quanto aos critérios de organização, conteúdo, atratividade e persuasão.

\begin{tabular}{ccc} 
Variáveis (Perguntas) & \multicolumn{2}{c}{ Respostas positivas } \\
\cline { 2 - 3 } A. Organização & N & $\%$ \\
\hline A. 1 A capa mostra o assunto do álbum? & 30 & 100 \\
A. 2 Você acha que o número de figuras do álbum está adequado? & 28 & 93,3 \\
A. 3 Você gostou das cores utilizadas no álbum? & 30 & 100 \\
\hline B. Conteúdo & & \\
\hline B.1 O álbum mostra informações sobre hábitos saudáveis? & 30 & 100 \\
\hline C. Atratividade & 30 & 100 \\
\hline C.1 A capa chamou sua atenção? & 30 & 100 \\
\hline D. Persuasão & 30 & 100 \\
\hline C.2 Você sente vontade de conversar sobre o álbum? & \multicolumn{2}{c}{} \\
\hline D.1 Você pretende seguir as orientações do álbum? & 29 & 96,7 \\
us.2 Se você tivesse que informar outra pessoa sobre como controlar o peso, & 29 & 96,7 \\
\hline
\end{tabular}

Fonte: Oliveira RA, et al., 2020.

Diante das respostas dos participantes, observou-se que todos os critérios avaliados obtiveram mais de $90 \%$ de respostas positivas. Os blocos conteúdo e atratividade foram os que apresentaram maior positividade nas respostas. 
O tópico "E" do questionário referia-se à aceitação cultural, no qual os pacientes puderam opinar o que gostaram e o que não gostaram do álbum. Em relação a esse tópico, $13(43,3 \%)$ referiram que gostaram das orientações e ilustrações contidas no álbum. As sugestões envolveram alterar o formato do sapato contido na figura 10 e alterar a figura 11 que trata da lesão no pé diabético. A seguir, relata-se a avaliação de cada figura do álbum feita pelos pacientes em relação à clareza (Gráfico 1).

Gráfico 1 - Avaliação das figuras quanto à clareza.

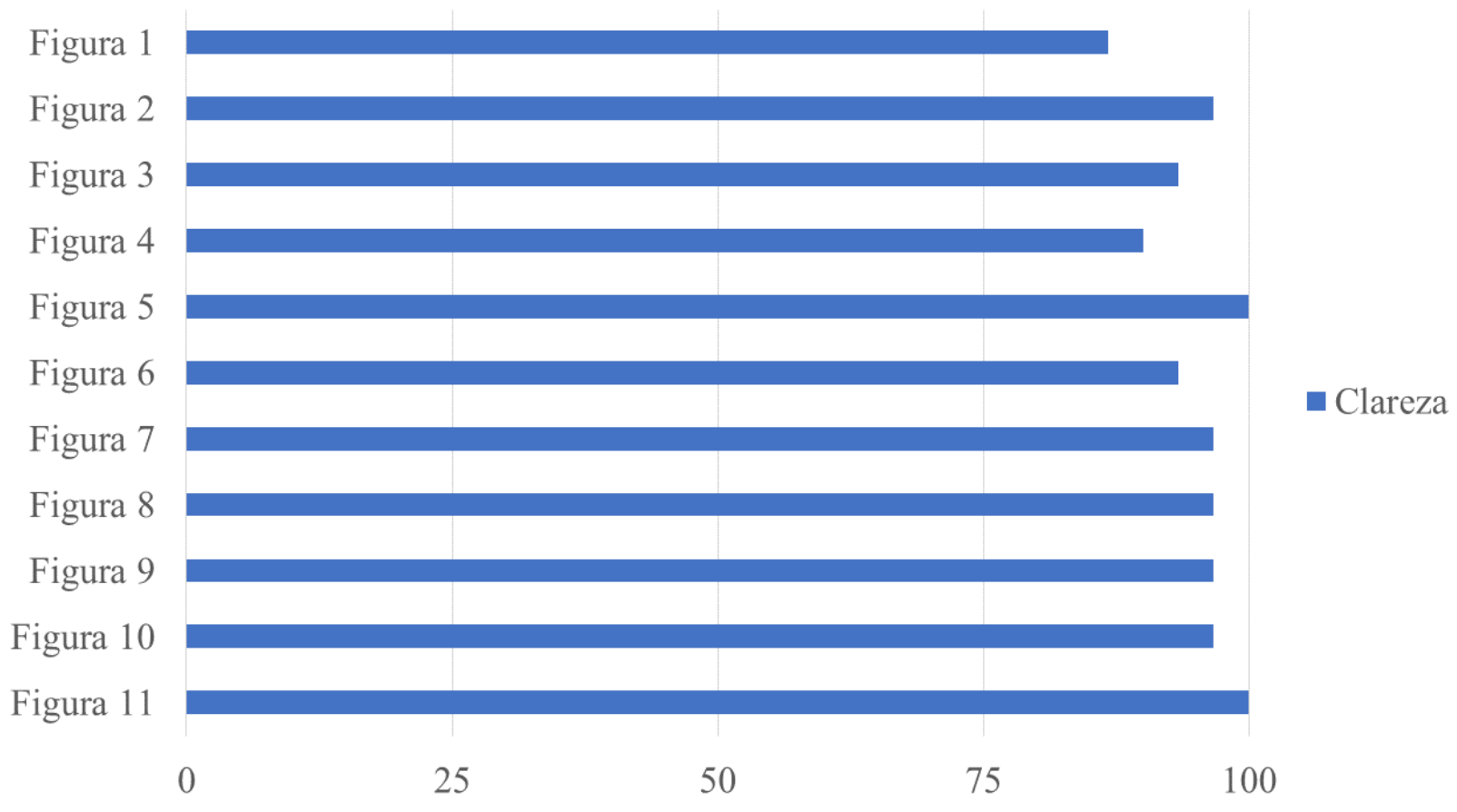

Fonte: Oliveira RA, et al., 2020.

Os pacientes avaliaram cada figura quanto à clareza (claro, pouco claro e confuso). As respostas positivas ("claro") a essa questão variaram de $86,7 \%$ a $100 \%$. A figura 1 foi a única que atingiu porcentagem de respostas positivas menor que $90 \%$. Já as figuras 5 e 11 foram consideradas claras por $100 \%$ dos pacientes. A seguir, apresenta-se os Índices de Validação do Conteúdo (IVC) de cada figura segundo a análise dos pacientes (Tabela 2).

Tabela 2 - Distribuição dos IVC de cada figura na percepção dos pacientes.

\begin{tabular}{cc}
\hline Figuras & IVC \\
\hline 1 & 1 \\
2 & 1 \\
3 & 0,96 \\
4 & 1 \\
5 & 0,96 \\
6 & 1 \\
7 & 1 \\
8 & 1 \\
9 & 0,96 \\
10 & 1 \\
11 & 1 \\
\hline Média & 0,98 \\
\hline
\end{tabular}

Fonte: Oliveira RA, et al., 2020. 
As figuras 3, 5 e 9 foram as únicas que apresentaram IVC menor que 1. A média global do IVC das figuras foi 0,98 . O IVC variou de 0,96 a 1 .

\section{DISCUSSÃO}

De acordo com a validação realizada com 30 pacientes com diabetes, o álbum seriado "De olho no pé diabético" foi considerado um material educativo que apresenta aparência e conteúdo válidos, uma vez que a tecnologia obteve os valores de IVC superiores a 0,80 .

Uma ferramenta de extrema importância para a realização do processo educativo nas práticas de educação em saúde tem sido a construção e validação de Tecnologias Educacionais (TE) assertivas, de fácil entendimento para o público alvo e que considerem as peculiaridades de cada faixa etária, estejam em uma linguagem clara em relação ao tema abordado, e que atentem aos aspectos sociodemográficos, culturais, bem como a opinião dos participantes do estudo.

As TE possuem uma vertente educacional e um dos exemplos desse tipo de tecnologia são as cartilhas, visto que são construídas para fortalecer a orientação aos familiares e pacientes, podendo contribuir favoravelmente no processo de comunicação, ilustração e orientação, visando aumentar a adesão ao tratamento e o poder de decisão (PAULA FMS, et al., 2017).

Nas características da amostra, encontrou-se que $73,3 \%$ dos entrevistados eram do sexo feminino e $26,7 \%$ do sexo masculino. Esse resultado está em concordância com os achados do estudo realizado em Salvador que encontrou $61,8 \%$ de mulheres (PALMEIRA CS e PINTO SR, 2015).

A maior frequência de mulheres portadoras de Diabetes Mellitus pode ser associada a vários fatores, dentre eles o fato de as mulheres serem mais atentas quanto aos sintomas e sinais físicos das doenças e procurarem assistência em serviços de saúde com maior frequência que os homens (MANSYUR CL, et al., 2015; SANTOS AD, et al., 2018). Entretanto, outros autores afirmam que o maior acometimento é no sexo masculino, como exemplo demonstrado no estudo em Alagoas, onde se evidenciou o predomínio leve de diabetes mellitus no sexo masculino (54,75\%) (SANTOS AD, et al., 2018). Assim, é possível perceber que não existe um consenso na literatura sobre qual gênero é o mais predominante.

A indiferenciação da prevalência do DM por sexo, todavia, não se reflete na elaboração de estratégias de intervenção e controle da doença, haja vista que seu desenvolvimento pode ocasionar consequências onerosas a saúde dos indivíduos independente do sexo. Faz-se necessário mais pesquisas para melhor compreender os aspectos epidemiológicos relacionados ao gênero.

A figura 1 foi a única que a porcentagem de respostas positivas em relação à clareza foi menor que $90 \%$. Esse número refere ao não entendimento da imagem que caracteriza um pâncreas saudável e um pâncreas não saudável, pois muitos indivíduos afirmaram não compreenderem a imagem do órgão pâncreas sinalizando os alimentos benéficos e maléficos ao paciente com diabetes mellitus, o que evidencia a importância dos resultados dessa avaliação, visto que o conhecimento da alimentação saudável, rica em frutas, legumes e verduras é considerado um dos fatores de proteção do desenvolvimento do pé diabético e suas complicações, sendo necessário o devido esclarecimento para os pacientes (BENTO LDF, et al., 2016).

O nível de compreensão pode ser relacionado ao nível de escolaridade, pois o período de 8 a 10 anos totalizou o maior quantitativo de pacientes, o que demonstra que o grau de escolaridade se coloca como um importante indicador socioeconômico para a compreensão do perfil dos usuários que são atendidos na unidade.

Uma baixa escolaridade também afeta a obtenção de recursos financeiros, refletindo na dificuldade de adquirir insumos para manutenção do tratamento e controle da doença. Tal associação já havia sido verificada anteriormente, tanto no Brasil (NASS EMA, et al., 2019) como em outros países (ISER BPM, et al., 2014).

As figuras 5 e 11 foram as que apresentaram os melhores índices de clareza, com 100\% de aprovação dos o que demonstra uma maior facilidade de entendimento. Essas figuras retratam as complicações do Diabetes Mellitus como a Hipoglicemia e o pé diabético, respectivamente. 
Considera-se o pé diabético uma das complicações mais graves da doença, visto que se estima que aproximadamente $15 \%$ dos indivíduos com DM vão desenvolver alguma lesão nos pés ao longo da vida e essa complicação é responsável por $40 \%$ a $60 \%$ dos casos de amputações dos membros inferiores (PALMEIRA CS e PINTO SR, 2015; SILVA PL, et al., 2015).

Esse fato demonstra a importância da abordagem educativa a esses pacientes com o intuito de minimizar os riscos a sua saúde. É de suma importância que essa abordagem seja realizada nos diversos serviços de saúde, por profissionais capacitados, que podem fazer uso de tecnologias confiáveis que facilitem esse processo.

Um estudo australiano realizado com trabalhadores de saúde aborígenes (AHWS) que avaliou a aceitabilidade na utilização de um álbum seriado sobre câncer, evidenciou que o recurso foi considerado útil, valioso e culturalmente apropriado por quase todos os profissionais de saúde.

Entretanto, a utilização da estratégia foi baixa devido a fatores como a falta de adequação do recurso para a realidade de alguns pacientes (BIERBAUM M, et al., 2017). Outro estudo realizado na Suécia mostrou que $29 \%$ dos materiais educativos fornecidos em 27 hospitais aos pacientes submetidos à cirurgia de câncer colorretal eram de difícil compreensão para o público-alvo (SMITH F, et al., 2014).

Dessa forma, evidencia-se que validar materiais educativos com representantes do público-alvo é uma atitude necessária para avaliar sua legibilidade, adequação e compreensibilidade, visto que considerar a opinião da população, que é o destino final da tecnologia, torna o álbum ainda mais eficaz em sua prática.

Concernente à validação do álbum seriado, observou-se que os índices de atratividade, organização, clareza e relevância foram considerados como satisfatórios pelo público abordado. Ao analisar a linguagem e as características do álbum seriado, observa-se que a comunicação foi considerada efetiva, visto que a transmissão da informação sobre a prevenção do pé diabético foi exitosa.

Dessa forma, pode-se demonstrar, por meio de uma análise quantitativa, a validação da tecnologia educativa como uma importante ferramenta para os atendimentos em saúde, seja na atenção básica em saúde ou até mesmo na atenção terciária.

No que diz respeito às limitações deste estudo, a coleta de dados ocorreu em ambientes ruidosos e com grande fluxo de pacientes, o que resultou em distração para os participantes. Além disso, foi relatada a preocupação de que o tempo destinado a pesquisa pudesse resultar em atraso nas consultas e atendimentos no centro especializado. Dessa forma, a compreensão das perguntas pode ter sido influenciada pelos fatores anteriormente citados.

\section{CONCLUSÃO}

O processo de validação clínica do álbum seriado "De olho na prevenção do pé diabético" possibilitou um retorno positivo do público-alvo aos idealizadores da tecnologia leve dura, demonstrando que o álbum é válido e pertinente para utilização em todos os aspectos analisados: organização, conteúdo, atratividade e persuasão, podendo, desse modo, ser utilizado não somente na atenção secundária, mas em todos os âmbitos de atenção à saúde. Foi percebido que as variáveis de conteúdo e atratividade foram avaliadas com maior pontuação, além de uma média global do IVC das figuras de 0,98 , fato que demonstra a boa aceitação e compreensão das imagens pelos pacientes entrevistados. Ressalta-se a importância do papel do enfermeiro nas estratégias de educação em saúde e orientação aos pacientes. Nesse contexto, o uso da tecnologia educativa apresenta-se como um importante instrumento de facilitação, fixação e bom entendimento por parte do público-alvo.

\section{REFERÊNCIAS}

1. AQUINO J, et al. Cartilha educativa sobre diabetes: elaboração e validação de conteúdo. Semina: Ciências Biológicas e da Saúde, 2016; 37(1): 77. 
2. BARBOSA EMG, et al. Educational technologies to encourage (self) care in postpartum women. Revista Brasileira de Enfermagem, 2016; 69(3): 545-553.

3. BENTO LDS, et al. A Perspectiva da vulnerabilidade na avaliação do pé diabético sob a ótica de enfermeiros. Cogitare Enferm, 2016; 21(1).

4. BOELL JEW, et al. Fatores de risco para o desencadeamento do pé diabético. Revista Eletrônica de Enfermagem, 2014; 16(2): 386-393.

5. BIERBAUM M, et al. Desafios para captação de recursos de educação sobre câncer por trabalhadores de saúde aborígenes rurais: a experiência do flipchart de Mensagens de Cura do Câncer. Saúde Rural e Remota, 2017; 17: 4199.

6. FEHRING R. Validating diagnostic labels: Standardized methodology. Classification of nursing diagnoses: Proceedings of the sixth conference, 1986; 183-190.

7. FONSECA KP, RACHED CD. Complicações do diabetes mellitus. International Journal of Health Management Review, 2019; 5(1): 1-13.

8. INTERNATIONAL DIABETES FEDERATION. IDF Diabetes Atlas. 8th ed. Brussels; 2017.

9. ISER BPM, et al. Prevalence, Correlates, and Description of Self-Reported Diabetes in Brazilian Capitals - Results from a Telephone Survey. Icks A, editor. PLoS ONE, 2014; 25;9(9): e108044.

10. LIMA ACMACC, et al. Construção e Validação de cartilha para prevenção da transmissão vertical do HIV. Acta Paulista de Enfermagem, 2017; 30(2): 181-189.

11. MANSYUR CL, et al. Social factors and barriers to self-care adherence in Hispanic men and women with diabetes. Patient Education and Counseling, 2015; 98(6):805-10.

12. NASS EMA, et al. Auto eficácia psicossocial em jovens com Diabetes Mellitus e sua influência no autocuidado. Rev Rene, 2019; 20:e41412.

13. PASQUALI L. Instrumentação psicológica: Fundamentos e práticas. 1aㅡ ed. Porto Alegre (RS): Artmed, 2010; 560p.

14. PALMEIRA CS, Pinto SR. Perfil epidemiológico de pacientes com diabetes mellitus em Salvador, Bahia, Brasil (20022012). Revista Baiana de Enfermagem, 2015 28;29(3):240.

15. PAULA FMS, et al. Elaboração de material didático para processamento de produtos para saúde em unidades de atenção primária à saúde. Revista SOBECC, 2017 ;22(3):170.

16. SABINO LMM, et al. Validação de cartilha para promoção da auto eficácia materna na prevenção da diarreia infantil. Revista Brasileira de Enfermagem, 2018; 71(3): 1412-1419.

17. SANTOS AD, et al. Epidemiological Profile of Patients with Diabetes Mellitus. Brazilian Journal Of Surgery And Clinical Research, 2018; 24(2): 40-46.

18. SANTOS CT. Tecnologias Voltadas Para educação Em Saúde: O Que Temos Para A Saúde Dos Idosos? Anais do Seminário Tecnologias Aplicadas à Educação e Saúde, 2015; 29-30: 14-21.

19. SILVA PL, et al. Cuidados com os pés: o conhecimento de indivíduos com diabetes mellitus cadastrados no programa saúde da família. Enferm. Glob, 2015; 37: 52-64.

20. SMITH F, et al. Readability, suitability and comprehensibility in patient education materials for Swedish patients with colorectal cancer undergoing elective surgery: A mixed method design. Patient Education and Counseling, 2014; 94(2):202-9. 